\title{
BMJ Open A review of rate control in atrial fibrillation, and the rationale and protocol for the RATE-AF trial
}

\author{
Dipak Kotecha, ${ }^{1,2,3,4}$ Melanie Calvert,, ${ }^{4,5}$ Jonathan J Deeks, ${ }^{5,6}$ Michael Griffith, ${ }^{2}$ \\ Paulus Kirchhof, ${ }^{1,2,3,4}$ Gregory YH Lip, ${ }^{1,3,4}$ Samir Mehta, ${ }^{6}$ Gemma Slinn, ${ }^{6}$ \\ Mary Stanbury, ${ }^{7}$ Richard P Steeds, ${ }^{1,2}$ Jonathan N Townend ${ }^{1,2}$
}

To cite: Kotecha D, Calvert M, Deeks JJ, et al. A review of rate control in atrial fibrillation, and the rationale and protocol for the RATE-AF trial. BMJ Open 2017;7:e015099. doi:10.1136/ bmjopen-2016-015099

- Prepublication history and additional material for this paper are available online. To view, please visit the journal online (http://dx.doi.org/10.1136/10. 1136/bmjopen-2016-015099)

DK is the Chief Investigator

Received 29 November 2016 Revised 29 April 2017 Accepted 23 May 2017

CrossMark

${ }^{1}$ Institute of Cardiovascular Sciences, University of Birmingham, Birmingham, UK

${ }^{2}$ Cardiology, University Hospitals Birmingham NHS Trust,

Birmingham, UK

${ }^{3}$ Cardiology, Sandwell \& West Birmingham Hospitals NHS

Trust, Birmingham, UK

${ }^{4}$ Centre for Patient Reported Outcomes Research, University of Birmingham, Birmingham, UK ${ }^{5}$ Institute of Applied Health

Research, University of

Birmingham, Birmingham, UK

${ }^{6}$ Birmingham Clinical Trials

Unit, University of Birmingham,

Birmingham, UK

${ }^{7}$ (Lead for the Patient and Public Involvement panel), Birmingham, UK

Correspondence to

Dr. Dipak Kotecha;

d.kotecha@bham.ac.uk

\section{ABSTRACT}

Background and objective Atrial fibrillation (AF) is common and causes impaired quality of life, an increased risk of stroke and death as well as frequent hospital admissions. The majority of patients with $\mathrm{AF}$ require control of heart rate. In this article, we summarise the limited evidence from clinical trials that guides prescription, and present the rationale and protocol for a new randomised trial. As rate control has not yet been shown to reduce mortality, there is a clear need to compare the impact of therapy on quality of life, cardiac function and exercise capacity. Such a trial should concentrate on the longterm effects of treatment in the largest proportion of patients with $A F$, those with symptomatic permanent $A F$, with the aim of improving patient well-being.

Design and intervention The RAte control Therapy Evaluation in permanent Atrial Fibrillation (RATE-AF) trial will enrol 160 participants with a prospective, randomised, open-label, blinded end point design comparing initial rate control with digoxin or bisoprolol. This will be the first head-to-head randomised trial of digoxin and betablockers in AF.

Participants Recruited patients will be aged $\geq 60$ years with permanent $A F$ and symptoms of breathlessness (equivalent to New York Heart Association class II or above), with few exclusion criteria to maximise generalisability to routine clinical practice.

Outcome measures The primary outcome is patientreported quality of life, with secondary outcomes including echocardiographic ventricular function, exercise capacity and biomarkers of cellular and clinical response. Follow-up will occur at 6 and 12 months, with feasibility components to inform the design of a future trial powered to detect a difference in hospital admission. The RATE-AF trial will underpin an integrated approach to management including biomarkers, functions and symptoms that will guide future research into optimal, personalised rate control in patients with AF. Ethics and dissemination East Midlands-Derby Research Ethics Committee (16/EM/0178); peer-reviewed publications.

Trial registration Clinicaltrials.gov: NCT02391337; ISRCTN: 95259705. Pre-results.

\section{INTRODUCTION}

Atrial fibrillation (AF) is a common cause of stroke and cardiovascular death, leads to poor quality of life and doubles the risk of

\section{Strengths and limitations of this study}

- Control of heart rate is universally used in patients with atrial fibrillation (AF), but evidence from good quality randomised controlled trials is extremely limited.

- Despite common clinical use, there has never been a direct randomised comparison of beta-blockers and digoxin for heart rate control in patients with $\mathrm{AF}$ (with or without heart failure).

- The RAte control Therapy Evaluation in permanent Atrial Fibrillation (RATE-AF) trial will assess the effect of therapy on patient-reported quality of life, and improve methods to capture this information in patients with AF. The trial will also evaluate the longterm impact on cardiac function, define reproducible methods to measure systolic and diastolic function in $\mathrm{AF}$ and develop new biomarkers for personalisation of treatment.

- The trial will not have the power to identify differences in clinical events, but will allow us to plan a future trial designed to detect a difference in the need for admissions to hospital.

hospital admission. ${ }^{1}$ We are currently in the midst of an epidemic of AF, with both incidence and prevalence expected to double in the next 20 years. ${ }^{2-4}$ Although AF can affect any age group, patients are typically elderly with significant comorbidities, including up to $50 \%$ suffering from heart failure. ${ }^{5} \mathrm{AF}$ is both a cause and consequence of heart failure, with complex interactions leading to impairment of systolic and diastolic function. ${ }^{67}$ The combination of these two conditions is expected to have a dramatic impact on the burden of healthcare worldwide. ${ }^{8-11}$

Management of AF involves anticoagulation to prevent strokes, selecting appropriate patients for restoration of sinus rhythm and almost universal need for control of heart rate. In contrast to other management strategies, the choice of rate 


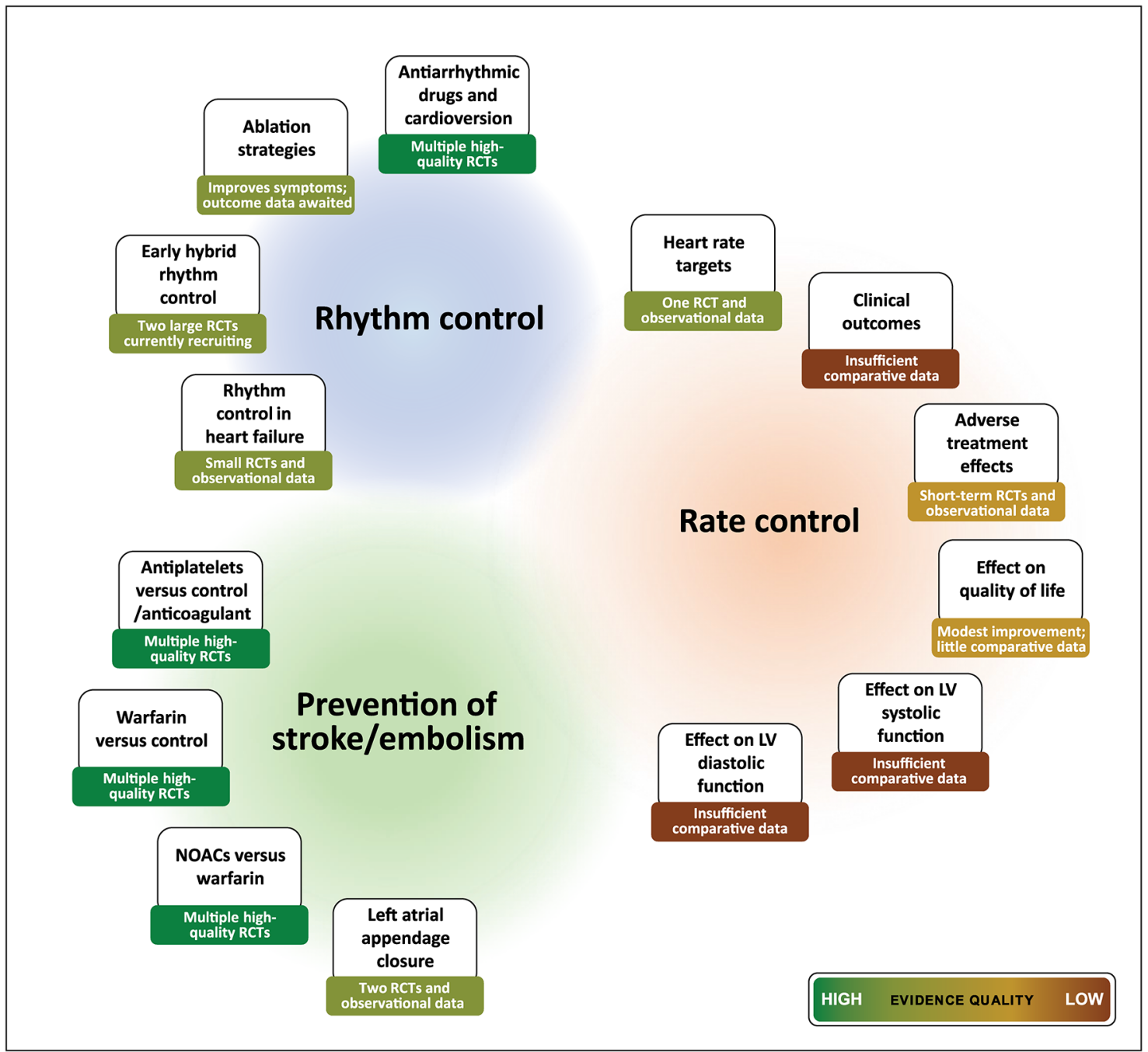

Figure 1 Evidence-based summary for management of atrial fibrillation. Summary of evidence for main components of clinical management, highlighting paucity of robust data for key issues regarding rate control therapy. RCT, randomised controlled trial; LV, left ventricular; NOAC, novel oral anticoagulants.

control therapy has a very low-quality evidence base (figure 1). ${ }^{12}$ Guidelines from the National Institute for Health and Care Excellence and the European Society of Cardiology (ESC) have mandated further research specifically on rate control, ${ }^{13}$ which is also reflected in the level of recommendations from the American Heart Association. ${ }^{14}$ The small studies currently available are often uncontrolled or with short follow-up, ${ }^{15-19}$ providing few insights on the biological effects of treatment or the mechanisms underpinning the response to therapy. With no evidence for any impact of rate control on mortality, ${ }^{20} 21$ and limited data for any difference in quality of life or functional outcomes, the choice of rate control agent is currently informed by expert consensus and physician experience.

In this paper, we review the current evidence-base for rate control in $\mathrm{AF}$ and the rationale for a new randomised controlled trial (RCT). The RAte control Therapy Evaluation in permanent Atrial Fibrillation (RATE-AF) trial will compare initial therapy with beta-blockers versus digoxin in older patients with symptomatic permanent
AF, assessing quality of life, functional capacity, left-ventricular ejection fraction (LVEF), diastolic function and biomarkers of treatment response.

\section{Rationale for a new trial of rate control in AF \\ Why not choose a rhythm control strategy?}

A number of RCTs have assessed the addition of rhythm control strategies to control of heart rate in patients with $\mathrm{AF}$, most often with anti-arrhythmic drugs (AAD) and direct current cardioversion. Neither of the two largest trials found any difference in clinical outcomes comparing these approaches (Atrial Fibrillation Follow-up Investigation of Rhythm Management (AFFIRM) and Rate Control versus Electrical Cardioversion for Persistent Atrial Fibrillation (RACE)).$^{22}{ }^{23}$ Other smaller trials and meta-analyses have confirmed that rhythm control is not superior to regulation of heart rate alone, ${ }^{24-26}$ including heart failure patients with both impaired and preserved ejection fraction. ${ }^{27} 28$ These studies have analysed heterogeneous populations, including both paroxysmal and permanent $\mathrm{AF}$ that may differ with regard to mechanism, 


\section{Hospitalisation: Rate vs rhythm-control}

Study

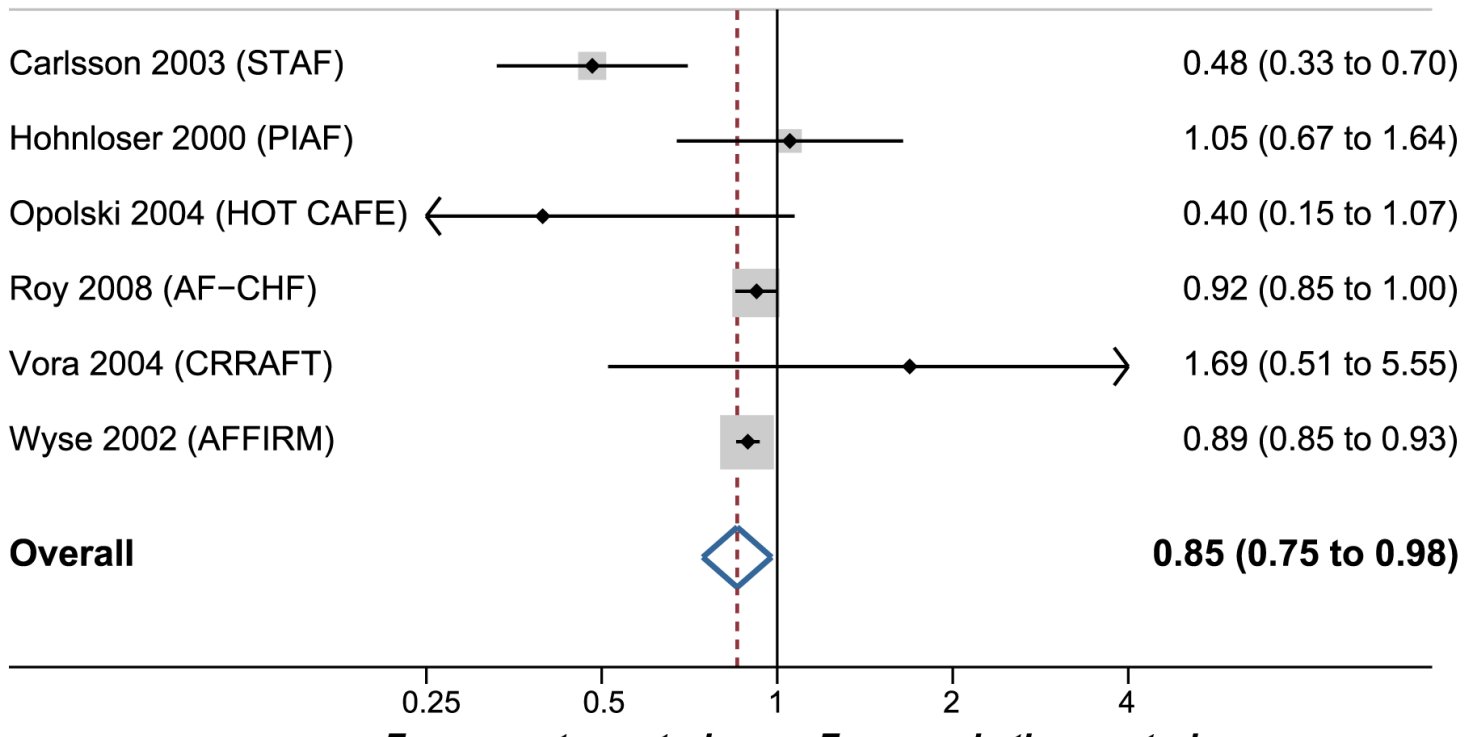

Favours rate-control
Risk ratio $(95 \% \mathrm{Cl})$

$0.48(0.33$ to 0.70$)$

1.05 (0.67 to 1.64$)$

0.40 (0.15 to 1.07$)$

$0.92(0.85$ to 1.00$)$

$1.69(0.51$ to 5.55$)$

$0.89(0.85$ to 0.93$)$

Favours rhythm-control

Figure 2 Hospitalisation in rate vs rhythm control trials. Meta-analysis of hospitalisation in the six largest rate vs rhythm control trials, excluding hospital visits for cardioversion procedures, where applicable. Studies are pooled with a random-effects model. Significant heterogeneity was identified, with an $\mathrm{I}^{2}$ value of $66.8 \%(\mathrm{p}=0.01)$. Grey boxes represent the comparative weight of the study. STAF, Strategies of Treatment of Atrial Fibrillation study (cardioversion/AAD vs rate control in persistent AF) ${ }^{76}$; PIAF, Pharmacological Intervention in Atrial Fibrillation trial (amiodarone/cardioversion vs diltiazem in persistent AF) ${ }^{77}$; HOT CAFE, How to Treat Chronic Atrial Fibrillation study (cardioversion/AAD vs rate control in persistent AF) ${ }^{78}$; AF-CHF, Atrial Fibrillation and Congestive Heart Failure trial (cardioversion/AAD vs rate control in paroxysmal/persistent AF with LVEF $\leq 35 \%)^{27}$; CRAAFT, Control of Rate vs Rhythm in rheumatic Atrial Fibrillation Trial (cardioversion/amiodarone vs diltiazem in persistent AF due to rheumatic heart disease) ${ }^{79}$; AFFIRM, Atrial Fibrillation Follow-up Investigation of Rhythm Management study (AAD/cardioversion versus rate control in paroxysmal/persistent AF); AAD, anti-arrhythmic drugs; LVEF, left-ventricular ejection fraction. ${ }^{22}$

prognosis and the response to treatment. ${ }^{15}$ However, there is also evidence that a rhythm control strategy may increase hospital admissions. A meta-analysis of major published trials is presented in figure 2, highlighting a $17 \%$ increase in the risk of hospitalisation in the rhythm control group (after exclusion of hospital visits related to cardioversion). Although limited by patient crossover and the association between $\mathrm{AAD}$ and adverse events, ${ }^{29}$ the results highlight the importance of trials comparing different rate control options and associated healthcare costs.

Although AF ablation is becoming increasingly popular, it remains a highly invasive method to restore sinus rhythm. ${ }^{30}{ }^{31}$ Current guidelines recommend ablation to improve AF-related symptoms in patients with paroxysmal $\mathrm{AF}$, or as a treatment option in symptomatic persistent $\mathrm{AF}$ that is refractory to other therapy ${ }^{114}$ Long-term outcome studies are awaited and need to be balanced against procedural complications and AF recurrence. Even in patients receiving intensive rhythm control therapy, rate control is often necessary to reduce symptoms during AF paroxysms. Furthermore, $40 \%-50 \%$ of patients with $\mathrm{AF}$ are deemed as unsuitable for rhythm control (permanent
AF), ${ }^{532}$ and are maintained on rate control therapy to reduce potential symptoms and avoid tachycardia that may worsen ventricular function. ${ }^{6}$ Patients with permanent $\mathrm{AF}$ have a higher residual risk of cardiovascular death, stroke or systemic embolism, despite anticoagulation. $^{33}$

What is the optimal heart rate target in AF?

There is clinical uncertainty about how to control heart rate and the intensity of rate-reduction. In the RACE II trial of 614 randomised patients with permanent AF, there were no benefits of strict ( $<80 \mathrm{bpm}$ at rest) compared with lenient rate control (resting heart rate $<110 \mathrm{bpm}$ ) over 3 years of follow-up. ${ }^{34}$ Although interpretation was limited by the narrow difference in heart rate between groups, lenient rate control was found to be non-inferior with an adjusted HR of 0.80 (90\% CI 0.55 to 1.17) for a wide composite of adverse clinical outcomes (12.9\%, compared with $14.9 \%$ in the strict control arm). In addition, there were no differences in symptoms or New York Heart Association (NYHA) class, ${ }^{34} 35$ and patients achieving strict rate control required more clinic visits. ${ }^{36}$ These findings are consistent with other trials, ${ }^{37-39}$ registries $^{32}$ and even 
randomised ${ }^{40}$ and observational ${ }^{41}$ cohorts in patients with concomitant heart failure, suggesting that intensity of heart rate control is not the key determinant of outcomes in $\mathrm{AF}$.

\section{Do outcomes vary with different rate control therapies?}

Medical therapy to achieve rate control in AF can be achieved with beta-blockers, digoxin and non-dihydropiridine calcium channel blockers (CCB; diltiazem or verapamil). ${ }^{1}$ Only a limited evidence-base is available to assist clinicians in choosing first-line and subsequent therapy, resulting in wide variations in clinical practice ${ }^{42-44}$ and frequent use of combination therapy. Guidelines suggest the choice of medication should be individualised, dependent on the presence of ongoing symptoms. ${ }^{14}$ However, these recommendations are based on low-quality trials and observational data, often with small numbers of participants and follow-up over a few weeks. ${ }^{16}$ There are no RCTs comparing long-term rate control options in AF.

Demonstrating any reduction in hard clinical outcomes with rate control has proved elusive. In patients with heart failure, reduced ejection fraction and concomitant $\mathrm{AF}$, an individual patient-level meta-analysis of double-blind RCT data has suggested that beta-blockers do not reduce all-cause mortality or hospital admissions compared with placebo, ${ }^{20}$ in contrast to the substantial benefit seen in sinus rhythm. ${ }^{45}$ Similarly, the use of digoxin was not associated with any increase, or reduction, in mortality in a comprehensive systematic review. ${ }^{21}$ This finding deviates from prior observational analyses which are confounded by the fact that sicker patients tend to receive digoxin more often, which can only be addressed within a randomised trial. Although digoxin is known to reduce hospital admissions in patients with heart failure and reduced ejection fraction in sinus rhythm, ${ }^{46}$ the impact in patients with $\mathrm{AF}$ is unknown.

If rate control has limited effect on mortality, what about evidence for a differential effect on other outcomes, such as functional capacity, cardiac function or quality of life? Beta-blockers are the most commonly used rate control agents and although they have a greater impact than digoxin on heart rate during exertion, there is no evidence that this results in better exercise capacity. ${ }^{17} 18$ 47-49 Beta-blockers were not associated with any improvement in arrhythmia-related symptoms in a small RCT of 60 low-risk patients with permanent AF, compared with diltiazem and verapamil which reduced the frequency of symptoms. ${ }^{50}$ Those in the beta-blocker group had a reduction in exercise capacity and increase in B-type natriuretic peptide (BNP) compared with those treated with CCB. ${ }^{51}$ Analysis of smaller trials comparing beta-blockers with CCB are inconsistent. ${ }^{17}$ Compared with verapamil or diltiazem, digoxin has less effect on heart rate but there is no consistent evidence for any difference in functional outcomes. ${ }^{17} 18474952$ Importantly, diltiazem and verapamil are usually avoided in patients with reduced ejection fraction due to the risk of adverse outcomes,${ }^{53-57}$ leaving only beta-blockers or digoxin as suitable therapy. Only a single RCT has been published comparing beta-blockers with digoxin in patients with $\mathrm{AF}$ and heart failure (mean LVEF 24\%, $\mathrm{n}=47$ ) ${ }^{58}$ Although there was a marginally significant improvement in LVEF with combined carvedilol/digoxin versus placebo/ digoxin, blinded withdrawal of digoxin then led to a deterioration in LVEF, accompanied by an increase in BNP. The direct effects of digoxin on LVEF and diastolic function have only been studied in patients with sinus rhythm, where digoxin increased LVEF by $3 \%-11 \%$ and improved diastolic filling. ${ }^{59-61}$ Magnesium has been shown to complement digoxin therapy to achieve lower ventricular rates in $\mathrm{AF}^{62}$ but is not in common use due to the availability of beta-blockers and CCB which are more potent agents for acute heart rate control. ${ }^{1}$ Although data on patient-reported quality of life are limited, ${ }^{6364}$ rate control has been associated with improved quality of life in trials assessing rate versus rhythm control. ${ }^{65-67}$ The mechanism by which rate control therapy mediates an increase in physical functioning and quality of life is unknown but conceivably due to improvements in LVEF and/or diastolic function.

In summary, rate control is an important part of treatment in all patients with AF but the evidence-base is poor, particularly in those with permanent $\mathrm{AF}$ who form the majority of patients in clinical practice. Rate control in AF is also subject to considerable, and poorly characterised individual variability in response, with limited information about the effects of therapy on cardiac function, quality of life and functional capacity.

\section{The RATE-AF trial}

The RATE-AF trial is the first head-to-head randomised assessment of beta-blockers versus digoxin as the initial rate control agent in patients with $\mathrm{AF}$. The trial has a prospective, randomised, open-label, investigator-blinded end point (PROBE) design, and is planned as an inclusive study that reflects and will have an important impact on clinical practice (box 1). The primary outcome is patient-reported quality of life using the Short Form (36) Health Survey (SF-36) physical component summary score at 6 months' post-randomisation. The major secondary outcomes are change in LVEF and diastolic function on echocardiography, functional capacity, global and AF-specific quality of life and cardiovascular biomarkers (box 2). A key objective of the trial is to improve the methods used for measuring quality of life in patients with $\mathrm{AF}$, as well as optimising the validity, reproducibility and acquisition of echocardiographic left-ventricular function. The RATE-AF trial will also act as a feasibility study to plan a future, event-driven clinical trial exploring the impact of different rate control strategies on cardiovascular events and unplanned hospital admissions. The study is sponsored by the University of Birmingham and funded by the National Institute for Health Research (NIHR), as part of a Career Development Fellowship awarded to the Chief Investigator (DK). 
Box 1 The RAte control Therapy Evaluation in permanent Atrial Fibrillation (RATE-AF) trial-information for patients

\begin{abstract}
About atrial fibrillation
Atrial fibrillation is a common heart condition that leads to an irregular and often rapid heart rate. Atrial fibrillation causes 1 in 4 strokes, and patients have frequent hospital admissions and a higher risk of dying. In addition, atrial fibrillation makes many patients feel unwell, with reduced quality of life.
\end{abstract}

\section{What is the purpose of the trial?}

Atrial fibrillation usually requires medication to control heart rate, but we currently do not know which medication is better for patients. The aim of this study is to find out which of the two treatments improves quality of life and the function of the heart, digoxin or bisoprolol (a beta-blocker).

\section{What will happen in the trial?}

The RATE-AF trial is designed to compare two approaches for control of heart rate, based on initial treatment with either digoxin or betablockers, medications which are commonly used by doctors. The main objective of the trial is to research the effects of treatment on quality of life in patients with atrial fibrillation. We will also test whether quality of life questionnaires respond to changes in symptoms experienced by patients, how we use ultrasound to look at the function of the heart, and develop new markers in the blood to personalise treatment.

\section{More information}

RATE-AF trial video: https://www.youtube.com/watch?v=40xe8AcVo0E or search 'rateaf' in YouTube.

Patient information (British Heart Foundation): https://www.bhf.org.uk/ heart-health/conditions/atrial-fibrillation.

\section{METHODS}

\section{Patients}

Inclusion criteria are patients aged 60 years or older with breathlessness (equivalent to NYHA class II or more) and permanent $\mathrm{AF}$, characterised as a physician decision for rate control with no plans for cardioversion, AAD or ablation therapy. Only limited exclusion criteria apply (figure 3), reflecting any clear requirements or contraindications for either beta-blockers or digoxin. As neither agent impacts on mortality in patients with heart failure ${ }^{20}{ }^{21}$ reduced LVEF is not an exclusion criterion. All patients are expected to be anticoagulated if appropriate, according to their clinical risk of stroke and thromboembolism.

\section{Study procedures and outcomes}

One hundred and sixty eligible patients in need of rate control will be invited to participate in the study from primary and secondary care across two major NHS Trusts in Birmingham, UK. The RATE-AF trial is managed by the Birmingham Clinical Trials Unit (University of Birmingham) and situated within the Birmingham NIHR/Wellcome Trust Clinical Research Facility.

Following written informed consent, participants will be randomised in a 1:1 ratio to either bisoprolol or digoxin therapy. Randomisation will be provided by a computer-generated minimisation algorithm to ensure balance between the treatment arms for baseline European Heart Rhythm Association class and gender. Allocation will be concealed until the patient has been recruited and consented, thereafter the trial will be open-label.

Baseline assessment procedures will include patient-reported quality of life questionnaires (table 1), 6 min walk distance, echocardiography and biomarker assessment. Participants will then receive study medication (bisoprolol 1.25-15 mg once daily or low-dose digoxin 62.5-250 $\mu \mathrm{g}$ once daily), with scheduled uptitration visits to attain a heart rate at rest of $\leq 100 \mathrm{bpm}$. This heart rate is in line with international recommendations ${ }^{1}$ and was chosen pragmatically to reflect the opinion of many cardiologists that tachycardia can lead to, or worsen, systolic and diastolic dysfunction. Ambulatory 24 hours ECG monitoring will be performed at the end of uptitration (unblinded). Investigator-blinded end points will be assessed at the interim (6-month) and final (12-month) visit, which include patient-reported quality of life, echocardiographic parameters of systolic and diastolic left-ventricular function and biomarker assessment (figure 3).

\section{Exploratory work and clinical practice improvement}

During the trial, qualitative research using focus groups and structured interviews will assess whether the quality of life questionnaires adequately and acceptably assess changes in symptom burden in a sample of patients from each treatment arm. We will also compare and contrast the generic and AF-specific questionnaires. The aim of this work is to improve the methods used for measuring patient-reported outcomes in $\mathrm{AF}$, and to address some of the limitations we have identified in published validation studies. ${ }^{6}$

Optimal acquisition of echocardiography in patients with AF will be determined by reproducibility studies, comparing repeated measures of systolic/diastolic function according to cardiac cycle length. The RATE-AF trial will address the evidence gaps we have identified in a systematic review of echocardiography in patients with $\mathrm{AF}^{69}$ and explore the diagnostic difficulty of categorising heart failure in the context of AF (particularly with preserved ejection fraction, where symptom classification is confounded and BNP levels are consistently raised due to $\mathrm{AF}^{7}$

Blood samples from participants will be analysed for the cellular effects of rate control, including intracellular sodium, calcium and endogenous cardiotonic steroids (CTS) using photometry in cultured human cardiomyocytes. This work will give mechanistic insight into the cellular response to beta-blockers and digoxin, identify novel markers of treatment effect and develop assays that are more robust than serum digoxin concentration (SDC) for determining individual patient dosage. SDC is an immunoassay known to be a poor marker of digoxin toxicity, ${ }^{70}$ which can cross-react with other targets ${ }^{71}$ (eg, endogenous CTS). Although SDC will be performed at 6 months follow-up and as required during the trial to advise clinicians on dose and avoid high digoxin levels, digoxin toxicity remains a clinical diagnosis at present. 
Box 2 Outcomes and objectives of the RAte control Therapy Evaluation in permanent Atrial Fibrillation (RATE-AF trial

\section{Primary outcome:}

Comparison of two strategies for rate control on patient-reported quality of life, based on initial use of digoxin vs beta-blocker therapy, with a predefined focus on physical well-being using the SF- 36 physical component summary at 6 months.

\section{Secondary outcomes:}

- Patient reported quality of life at 6 and 12 months, including SF-36 global and domain-specific scores, EQ-5D-5L summary index and visual analogue scale, and AFEQT overall score.

- Echocardiographic left-ventricular function at 12 months, including LVEF and diastolic function (E/e' and composite of diastolic indices).

- Functional assessment at 6 and 12 months, including 6 min walking distance and change in EHRA class.

- Change in NT-proBNP levels at 6 months.

- Change in heart rate from baseline and group comparison using 24 hours ambulatory ECG at end of uptitration.

\section{Feasibility assessment:}

- Successful methods for recruitment across primary and secondary care.

- Key issues that affect retention of participants, such as convenience, compliance and cross-over.

- Drug discontinuation rate and adverse reactions leading to drug discontinuation.

- Therapy-induced requirement for additional treatment (eg, pacemaker implantation).

- Population-specific SD and proportions to enable sample size calculation for a future trial.

- Assessment of unplanned hospital admissions and cardiovascular outcomes.

\section{Exploratory objectives:}

- Assessment of the validity and reproducibility of echocardiographic measures in patients with AF.

- Correlation of baseline measures, including quality of life questionnaires and unblinded baseline investigations such as quality of life, NT-proBNP, LVEF, E/e', EHRA class, intracellular biomarkers and heart rate.

- Impact of therapy on intracellular sodium and calcium concentration and cardiotonic steroid levels as biomarkers of cellular response.

- Impact of combination therapy on outcomes.

- Change in cognitive function at 12 months.

- Qualitative research of patient-reported quality of life using focus groups to explore patient acceptability, optimal delivery methods and responsiveness.

- Correlation of serum digoxin concentration with change in quality of life and intracellular methods.

- Cost-consequence economic analysis from an NHS healthcare perspective.

AF, atrial fibrillation; AFEQT, Atrial Fibrillation Effect on QualiTy of life questionnaire; NT-proBNP, N-terminal pro B-type natriuretic peptide; EHRA, European Heart Rhythm Association functional class; EQ-5D-5L, EuroQol five dimensions five level questionnaire; LVEF, left ventricular ejection fraction; NHS, National Health Service; SF-36, Short Form (36) Health Survey.

Serum will also be stored for the development of new blood-based and genetic biomarkers that aid in personalisation of rate control therapy.

\section{Statistical considerations}

The null hypothesis is of no difference in the physical functioning domain of the SF-36 quality of life questionnaire when comparing a strategy of digoxin versus beta-blocker therapy for initial rate control in older patients with permanent AF. The alternative hypothesis is superiority of one over the other therapy as an initial strategy of care. Randomising 144 patients we can assume an $85 \%$ power to detect an effect size of half a SD in a continuous outcome measure of quality of life (two-sided $\alpha$ of 0.05 ). Assuming that $10 \%$ of patients will be lost to follow-up, 160 patients are needed. There is some evidence from existing research to support the notion that the treatment effect could be this large. This includes a $17 \%$ improvement in SF-36 role-physical score in the rate control arm of the RACE study, ${ }^{66}$ a $22 \%$ improvement in a proprietary symptom-checklist with CCB (compared with $8 \%$ change in those assigned beta-blockers), ${ }^{19}$ and $17 \%$ improvement with rate control using SF-36 in the PIAF trial. ${ }^{67}$ The RATE-AF trial will also help us to explore surrogates for clinical outcomes, such as LVEF using echocardiography and BNP, and provide estimates for a future definitive trial of rate control in AF, including reliable information on recruitment rates, study drug titration, cross-over, retention and healthcare costs.

\section{Trial oversight, management and registration}

RATE-AF will be conducted in accordance with guidelines for Good Clinical Practice (GCP) and the Declaration of Helsinki, and has regulatory approval from the Medicines and Healthcare products Regulatory Agency.

Oversight will be provided by a Trial Steering Committee, comprising an independent Data Monitoring Committee and members of the RATE-AF Trial Management Group. This includes representatives of the patient and public involvement panel, involved in both the design and management of the trial. A Clinical Events Committee will be formed to adjudicate on adverse events.

The RATE-AF trial is registered at Clinicaltrials.gov (NCT02391337), ISRCTN (95259705) and EudraCT (2015-005043-13). Further information can be obtained from the trial website, http://www.birmingham.ac.uk/ rate-af, and the trial protocol (see online supplementary material). The protocol was developed in accordance with the Standard Protocol Items for Randomized Trials 


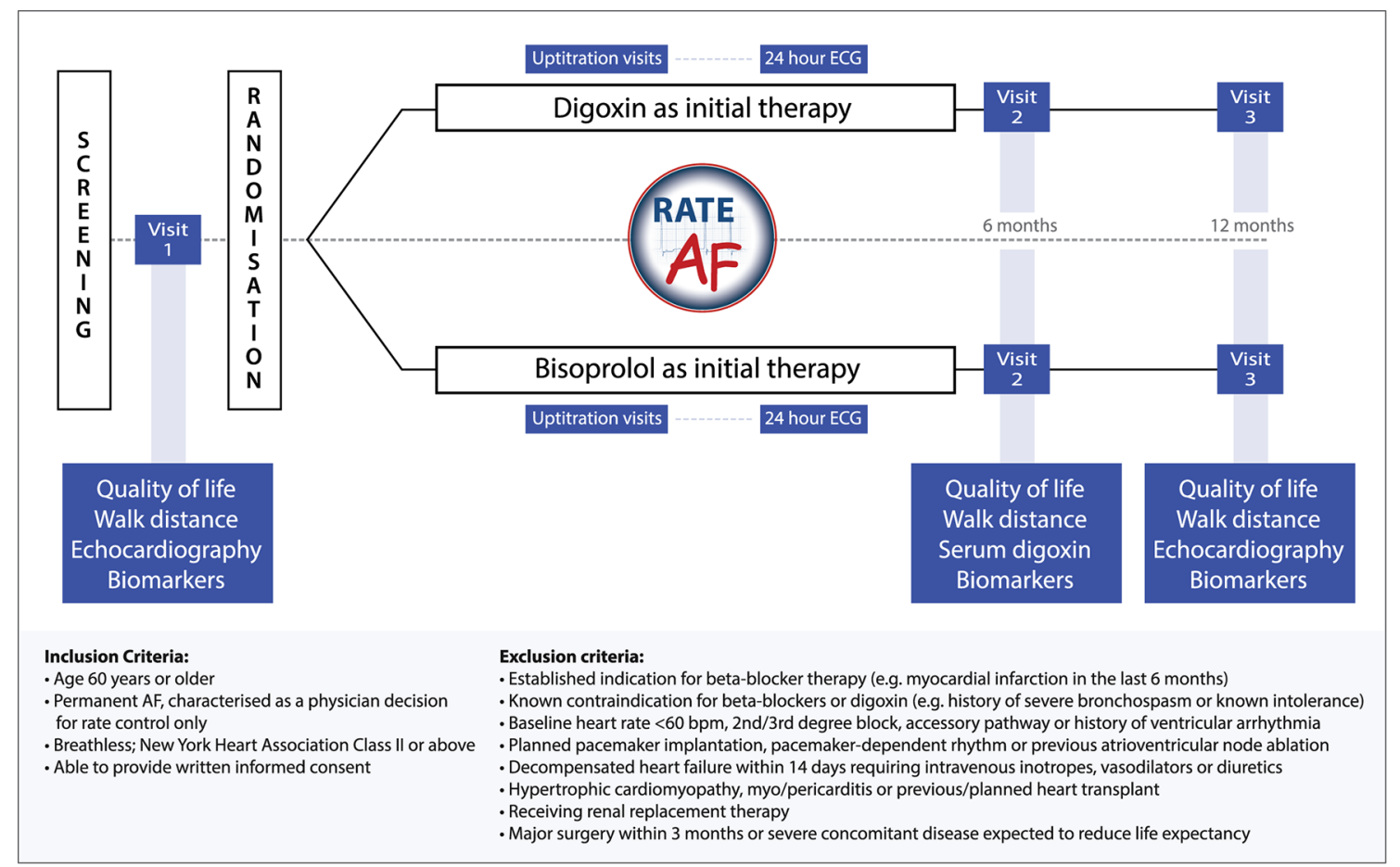

Figure 3 The RAte control Therapy Evaluation in permanent Atrial Fibrillation (RATE-AF) trial schema. Trial flow chart, including major end points and inclusion/exclusion criteria.

Table 1 Patient-reported quality of life questionnaires used in RAte control Therapy Evaluation in permanent Atrial Fibrillation

\section{Questionnaire Details \\ Short Form (36) Generic instrument with 4-week recall period in eights \\ Health Survey ${ }^{80}$ domains (vitality, physical functioning, bodily pain, general health perceptions, physical role functioning, emotional role functioning, social role functioning and mental health) \\ 11 subdivided questions, each recorded with a Likert scale \\ Scoring: each response is given a numerical value (0- \\ 100 , with 100 representing the best level of functioning \\ possible), which are averaged across each domain}

EuroQol five

Generic instrument about today's health with a five-

dimensions

five level

questionnaire 8283 answer scale in five domains (mobility, self-care, usual activities, pain/discomfort and anxiety/depression)

Scoring: each question is scored (1-5, with 1 representing the best health). The overall profile can be indexed to country-specific value sets giving a continuous value

Also includes a visual analogue scale denoting current health perception (0-100 scale, with 100 representing the best health the patient can imagine)

$\begin{array}{ll}\text { Atrial Fibrillation } & \text { AF-specific quality of life instrument with 4-week recall } \\ \text { Effect on } & \text { period in domains relating to symptoms, daily activities } \\ \text { QualiTy of life } & \text { and treatment } \\ \text { questionnaire }^{85} & \begin{array}{l}20 \text { questions (18 on health-related quality and life and } 2 \\ \text { on treatment satisfaction), each recorded with a 7-point } \\ \text { Likert scale } \\ \text { Scoring: responses to the } 18 \text { questions are summed } \\ \text { and converted to a continuous score (0-100, with 100 } \\ \text { corresponding to no patient concerns nor disability due } \\ \text { to AF) Component domains are scored in a similar way }\end{array}\end{array}$

\section{Advantages and disadvantages}

Extensively validated across a wide variety of conditions and the elderly ${ }^{81}$

Not specific to AF and hence other comorbidities may dominate responses

Requires a licence fee

Simple questionnaire that is quick to complete and includes a visual scale

Extensive utilisation, particularly for heath economic assessment, with improvement discrimination over prior versions ${ }^{84}$

Not specific to AF and hence other comorbidities may dominate responses

Specific to the impact of AF on quality of life Better than other AF-specific tools in a systematic review of methodological/ psychometric assessment ${ }^{68}$

Limited validation as yet in comparison with generic tools, ${ }^{86} 87$ particularly for clinical responsiveness Licence fee may apply 
statement, ${ }^{72}$ and the latest guidance from the International Society for Quality of Life Research Best Practice taskforce. $^{73-75}$

\section{Ethics and dissemination}

The trial has ethical approval from the East MidlandsDerby Research Ethics Committee (16/EM/0178) and approval from the National Health Service Health Research Authority (IRAS project ID: 191437).

The research findings will be submitted for publication to peer-reviewed journals after review by the oversight committees and the Patient Involvement Panel, and presented to relevant national and international meetings. Trial participants will be sent a lay summary of the final results of the trial, written by the Patient Involvement Panel.

\section{CONCLUSION}

Defining appropriate rate control therapy is vital, particularly in the rapidly growing number of older patients with permanent $\mathrm{AF}$, where current evidence is extremely limited. Rate control is an integral part of management in almost all patients with AF, but hardly any controlled trial evidence exists to guide the choice of agents. This is unacceptable in light of the potential benefits and possible adverse effects of treatment. In addition, the complete lack of data on the impact of medical therapy on symptom burden and heart function necessitate a programme of reproducibility and validity of both patient-reported quality of life and cardiac imaging in AF. The RATE-AF trial will answer key clinical questions about how to initiate therapy in order to improve patient well-being, stratified by relevant patient characteristics such as baseline symptoms, systolic and diastolic cardiac function, and biomarkers of treatment effect.

Correction notice This article has been corrected since it first published. The acronym for the Chief Investigator has been corrected. The Funding information has been updated with the correct ID of the Career Development Fellowship. Other typos and encoding errors have been corrected in the abstract, main text and reference list.

Acknowledgements We would like to acknowledge other members of the wider RAte control Therapy Evaluation in permanent Atrial Fibrillation team, including Karina Bunting, Patience Domingos, Dannie Fobian, Margaret Grant, Emma Hayes, Hannah Lack, Susan Jowett, Jonathan Mathers and Davor Pavlovic (University of Birmingham). We are indebted to the independent members of the trial oversight committees, as well as the Patient and Public Involvement Team.

Contributors The manuscript was drafted by DK who is the Chief Investigator for the RATE-AF trial. MG and GYHL are Principal Investigators. MC, PK, RPS and JNT are members of the Trial Management Group. JJD, SM and GS are representatives from the Clinical Trials Unit. MS is the Lead for the Patient Involvement Panel, and a member of the Steering Committee. All authors contributed to the writing of the RATE-AF protocol or patient information, and edited this manuscript for intellectual content.

Funding DK and the RATE-AF trial are supported by the National Institute of Health Research (NIHR) as part of a Career Development Fellowship (CDF-2015-08-074). The opinions expressed in this paper are those of the authors and do not represent the NIHR or the UK Department of Health.

Competing interests None of the authors report a conflict of interest. All authors have completed the ICMJE uniform disclosure form (www.icmje.org/ coi_disclosure.pdf) and declare: DK reports grants from Menarini, during the conduct of the study; non-financial support from Daiichi Sankyo and personal fees from AtriCure, outside the submitted work. MC reports grants from the National Institute of Health Research, during the conduct of the study; and personal fees from Astella Pharma and Ferring Pharma, outside the submitted work. PK reports consulting fees and honoraria from Bayer Healthcare, Boehringer Ingelheim, Bristol-Myers Squibb, Daiichi Sankyo, Medtronic, Pfizer and Servier, all outside the submitted work; research grants from Bristol-Myers Squibb, Pfizer, Cardiovascular Therapeutics, Daiichi Sankyo, Sanofi, St. Jude Medical, German Federal Ministry for Education and Research (BMBF), Fondation Leducq, German Research Foundation (DFG), European Union, British Heart Foundation and Medical Research Council UK, all outside the submitted work; and is listed on two patent applications on AF therapy and markers for AF, both outside the submitted work. GYHL has served as a consultant for Bayer, Astellas, Merck, AstraZeneca, Sanofi, BMS/ Pfizer, Biotronik, Portola and Boehringer Ingelheim, and has been on the speaker's bureau for Bayer, BMS/Pfizer, Boehringer Ingelheim and Sanofi Aventis. RPS is the President of the British Society of Echocardiography. JJD, MG, MS. JNT, SM, GS report no competing interests.

Patient consent Informed written consent was obtained from all trial participants using HRA and ethics-approved consent forms.

Ethics approval East Midlands-Derby Research Ethics Committee (16/EM/0178).

Provenance and peer review Not commissioned; externally peer reviewed.

Open Access This is an Open Access article distributed in accordance with the terms of the Creative Commons Attribution (CC BY 4.0) license, which permits others to distribute, remix, adapt and build upon this work, for commercial use, provided the original work is properly cited. See: http://creativecommons.org/ licenses/by/4.0/

C Article author(s) (or their employer(s) unless otherwise stated in the text of the article) 2017. All rights reserved. No commercial use is permitted unless otherwise expressly granted.

\section{REFERENCES}

1. Kirchhof P, Benussi S, Kotecha D, et al. 2016 ESC Guidelines for the management of atrial fibrillation developed in collaboration with EACTS. Eur Heart J 2016;37:2893-962.

2. Chugh SS, Havmoeller R, Narayanan K, et al. Worldwide epidemiology of atrial fibrillation: a Global Burden of Disease 2010 Study. Circulation 2014;129:837-47.

3. Krijthe BP, Kunst A, Benjamin EJ, et al. Projections on the number of individuals with atrial fibrillation in the European Union, from 2000 to 2060. Eur Heart J 2013;34:2746-51.

4. Lane DA, Skjøth F, Lip GYH, et al. Temporal Trends in Incidence, Prevalence, and Mortality of Atrial Fibrillation in Primary Care. J Am Heart Assoc 2017;6:e005155.

5. Chiang CE, Naditch-Brûlé L, Murin J, et al. Distribution and risk profile of paroxysmal, persistent, and permanent atrial fibrillation in routine clinical practice: insight from the real-life global survey evaluating patients with atrial fibrillation international registry. Circ Arrhythm Electrophysiol 2012;5:632-9.

6. Kotecha D, Piccini JP. Atrial fibrillation in heart failure: what should we do? Eur Heart J 2015;36:3250-7.

7. Kotecha D, Lam CS, Van Veldhuisen DJ, et al. Heart Failure With Preserved Ejection Fraction and Atrial Fibrillation: Vicious Twins. J Am Coll Cardiol 2016;68:2217-28.

8. Kotecha D, Banerjee A, Lip GY. Increased stroke risk in atrial fibrillation patients with heart failure: does ejection fraction matter? Stroke 2015;46:608-9.

9. Christiansen CB, Olesen JB, Gislason G, et al. Cardiovascular and non-cardiovascular hospital admissions associated with atrial fibrillation: a danish nationwide, retrospective cohort study. BMJ Open 2013;3:e001800.

10. Ambrosy AP, Fonarow GC, Butler J, et al. The global health and economic burden of hospitalizations for heart failure: lessons learned from hospitalized heart failure registries. J Am Coll Cardiol 2014;63:1123-33.

11. Kotecha D, Chudasama R, Lane DA, et al. Atrial fibrillation and heart failure due to reduced versus preserved ejection fraction: a systematic review and meta-analysis of death and adverse outcomes. Int J Cardiol 2016;203:660-6.

12. Kirchhof $P$, Breithardt $G$, Bax J, et al. A roadmap to improve the quality of atrial fibrillation management: proceedings from the fifth Atrial Fibrillation Network/European Heart Rhythm Association consensus conference. Europace 2016;18:37-50. 
13. National Institute for Health and Care Excellence. Atrial fibrillation: the management of atrial fibrillation. NICE clinical guideline 2014;180 http://www.nice.org.uk/guidance/cg180/.

14. January CT, Wann LS, Alpert JS, et al. ACC/AHA Task Force Members. 2014 AHA/ACC/HRS guideline for the management of patients with atrial fibrillation: executive summary: a report of the American College of Cardiology/American Heart Association Task Force on practice guidelines and the Heart Rhythm Society. Circulation 2014;130:2071-104.

15. Kotecha D, Kirchhof P. Rate and rhythm control have comparable effects on mortality and stroke in atrial fibrillation but better data are needed. Evid Based Med 2014;19:222-3.

16. Segal JB, McNamara RL, Miller MR, et al. The evidence regarding the drugs used for ventricular rate control. J Fam Pract 2000;49::47-59.

17. Nikolaidou T, Channer KS. Chronic atrial fibrillation: a systematic review of medical heart rate control management. Postgrad Med $\mathrm{J}$ 2009;85:303-12.

18. Farshi R, Kistner D, Sarma JS, et al. Ventricular rate control in chronic atrial fibrillation during daily activity and programmed exercise: a crossover open-label study of five drug regimens. J Am Coll Cardiol 1999;33:304-10.

19. Ulimoen SR, Enger S, Carlson J, et al. Comparison of four single-drug regimens on ventricular rate and arrhythmia-related symptoms in patients with permanent atrial fibrillation. Am J Cardiol 2013;111:225-30.

20. Kotecha D, Holmes $\mathrm{J}$, Krum H, et al. Efficacy of $\beta$ blockers in patients with heart failure plus atrial fibrillation: an individual-patient data meta-analysis. Lancet 2014;384:2235-43.

21. Ziff OJ, Lane DA, Samra M, et al. Safety and efficacy of digoxin: systematic review and meta-analysis of observational and controlled trial data. BMJ 2015;351:h4451

22. Wyse DG, Waldo AL, DiMarco JP, et al. A comparison of rate control and rhythm control in patients with atrial fibrillation. N Engl J Med 2002;347:1825-33.

23. Van Gelder IC, Hagens VE, Bosker HA, et al. A Comparison of Rate Control and Rhythm Control in Patients with Recurrent Persistent Atrial Fibrillation. N Engl J Med 2002;347:1834-40.

24. de Denus S, Sanoski CA, Carlsson J, et al. Rate vs rhythm control in patients with atrial fibrillation: a meta-analysis. Arch Intern Med 2005;165:258-62.

25. Chatterjee S, Sardar P, Lichstein E, et al. Pharmacologic rate versus rhythm-control strategies in atrial fibrillation: an updated comprehensive review and meta-analysis. Pacing Clin Electrophysiol 2013;36:122-33.

26. Al-Khatib SM, Allen LaPointe NM, Chatterjee R, et al. Rate- and rhythm-control therapies in patients with atrial fibrillation: a systematic review. Ann Intern Med 2014;160:760-73.

27. Roy D, Talajic M, Nattel S, et al. Rhythm control versus rate control for atrial fibrillation and heart failure. N Engl J Med 2008;358:2667-77

28. Kong MH, Shaw LK, O'Connor $\mathrm{C}$, et al. Is rhythm-control superior to rate-control in patients with atrial fibrillation and diastolic heart failure? Ann Noninvasive Electrocardiol 2010;15:209-17.

29. Corley SD, Epstein AE, DiMarco JP, et al. Relationships between sinus rhythm, treatment, and survival in the Atrial Fibrillation FollowUp investigation of Rhythm Management (AFFIRM) Study. Circulation 2004;109:1509-13.

30. Wazni O, Wilkoff B, Saliba W. Catheter ablation for atrial fibrillation. $N$ Engl J Med 2011;365:2296-304.

31. Jones DG, Haldar SK, Hussain W, et al. A randomized trial to assess catheter ablation versus rate control in the management of persistent atrial fibrillation in heart failure. J Am Coll Cardiol 2013;61:1894-903.

32. Kirchhof $\mathrm{P}, \mathrm{Ammentorp} \mathrm{B}$, Darius $\mathrm{H}$, et al. Management of atrial fibrillation in seven European countries after the publication of the 2010 ESC Guidelines on atrial fibrillation: primary results of the PREvention oF thromboemolic events--European Registry in Atrial Fibrillation (PREFER in AF). Europace 2014;16:6-14.

33. Senoo K, Lip GY, Lane DA, et al. Residual Risk of Stroke and Death in Anticoagulated Patients According to the Type of Atrial Fibrillation: AMADEUS Trial. Stroke 2015;46:2523-8.

34. Van Gelder IC, Groenveld HF, Crijns HJ, et al. Residual risk of stroke and death in anticoagulated patients according to the type of atrial fibrillation: AMADEUS Trial. N Engl J Med 2010;362:1363-73.

35. Groenveld HF, Crijns HJ, Van den Berg MP, et al. The effect of rate control on quality of life in patients with permanent atrial fibrillation data from the RACE II (Rate Control Efficacy in Permanent Atrial Fibrillation II) study. J Am Coll Cardiol 2011:58:1795-803.

36. Groenveld HF, Tijssen JG, Crijns HJ, et al. Rate control efficacy in permanent atrial fibrillation: successful and failed strict rate control against a background of lenient rate control: data from RACE II (Rate
Control Efficacy in Permanent Atrial Fibrillation). J Am Coll Cardiol 2013;61:741-8.

37. Van Gelder IC, Wyse DG, Chandler ML, et al. Does intensity of ratecontrol influence outcome in atrial fibrillation? an analysis of pooled data from the RACE and AFFIRM studies. Europace 2006;8:935-42.

38. Cooper HA, Bloomfield DA, Bush DE, et al. Relation between achieved heart rate and outcomes in patients with atrial fibrillation (from the Atrial Fibrillation Follow-up investigation of Rhythm Management [AFFIRM] Study). Am J Cardiol 2004;93:1247-53.

39. Groenveld HF, Crijns HJ, Rienstra M, et al. Does intensity of rate control influence outcome in persistent atrial fibrillation? data of the RACE study. Am Heart J 2009;158:785-91.

40. Kotecha D, Flather MD, Altman DG, et al. Heart Rate, Heart Rhythm, and Prognostic Benefits of Beta-Blockers in Heart Failure: Individual Patient-Data Meta-Analysis. J Am Coll Cardiol 2017.

41. Cullington D, Goode KM, Zhang J, et al. Is heart rate important for patients with heart failure in atrial fibrillation? JACC Heart Fail 2014;2:213-20

42. Steg PG, Alam S, Chiang CE, et al. Symptoms, functional status and quality of life in patients with controlled and uncontrolled atrial fibrillation: data from the RealiseAF cross-sectional international registry. Heart 2012;98:195-201.

43. Nabauer M, Gerth A, Limbourg T, et al. The registry of the german competence NETwork on Atrial Fibrillation: patient characteristics and initial management. Europace 2009;11:423-34.

44. Lip GY, Laroche C, Dan GA, et al. A prospective survey in european Society of Cardiology member countries of atrial fibrillation management: baseline results of EURObservational Research Programme Atrial Fibrillation (EORP-AF) Pilot General Registry. Europace 2014;16:308-19.

45. Kotecha D, Manzano L, Krum H, et al. Beta-Blockers in Heart Failure Collaborative Group. Effect of age and sex on efficacy and tolerability of $\beta$ blockers in patients with heart failure with reduced ejection fraction: individual patient data meta-analysis. BMJ 2016;353:i1855

46. Digitalis Investigation Group. The effect of digoxin on mortality and morbidity in patients with heart failure. $N$ Engl J Med 1997;336:525-33.

47. Koh KK, Kwon KS, Park HB, et al. Efficacy and safety of digoxin alone and in combination with low-dose diltiazem or betaxolol to control ventricular rate in chronic atrial fibrillation. Am J Cardiol 1995;75:88-90.

48. Lewis RV, McMurray J, McDevitt DG. Effects of atenolol, verapamil, and xamoterol on heart rate and exercise tolerance in digitalised patients with chronic atrial fibrillation. J Cardiovasc Pharmacol 1989;13:1-15.

49. Tsuneda T, Yamashita T, Fukunami M, et al. Rate control and quality of life in patients with permanent atrial fibrillation: the Quality of Life and Atrial Fibrillation (QOLAF) Study. Circ J 2006;70:965-70.

50. Ulimoen SR, Enger S, Carlson J, et al. Comparison of four single-drug regimens on ventricular rate and arrhythmia-related symptoms in patients with permanent atrial fibrillation. Am J Cardiol 2013;111:225-30.

51. Ulimoen SR, Enger S, Pripp AH, et al. Calcium channel blockers improve exercise capacity and reduce N-terminal Pro-B-type natriuretic peptide levels compared with beta-blockers in patients with permanent atrial fibrillation. Eur Heart J 2014;35:517-24.

52. Lewis RV, Irvine N, McDevitt DG. Relationships between heart rate, exercise tolerance and cardiac output in atrial fibrillation: the effects of treatment with digoxin, verapamil and diltiazem. Eur Heart $J$ 1988;9:777-81

53. McMurray JJ, Adamopoulos S, Anker SD, et al. ESC Guidelines for the diagnosis and treatment of acute and chronic heart failure 2012: The Task Force for the Diagnosis and Treatment of Acute and Chronic Heart Failure 2012 of the European Society of Cardiology. Developed in collaboration with the Heart Failure Association (HFA) of the ESC. Eur Heart J 2012;33:1787-847.

54. Elkayam U. Calcium channel blockers in heart failure. Cardiology 1998;89(Suppl 1):38-46.

55. Multicenter Diltiazem Postinfarction Trial Research Group. The effect of diltiazem on mortality and reinfarction after myocardial infarction. N Engl J Med 1988;319:385-92.

56. Goldstein RE, Boccuzzi SJ, Cruess D, et al. Diltiazem increases lateonset congestive heart failure in postinfarction patients with early reduction in ejection fraction. The Adverse Experience Committee; and the Multicenter Diltiazem Postinfarction Research Group. Circulation 1991:83:52-60.

57. The Danish Study Group on Verapamil in Myocardial Infarction. Secondary prevention with verapamil after myocardial infarction. Am J Cardiol 1990;66:33-40. 
58. Khand AU, Rankin AC, Martin W, et al. Carvedilol alone or in combination with digoxin for the management of atrial fibrillation in patients with heart failure? J Am Coll Cardiol 2003;42:1944-51.

59. Partanen J, Heikkilä J, Pellinen T, et al. Effect of digoxin on the heart in normal subjects: influence of isometric exercise and autonomic blockade: a noninvasive study. Br J Clin Pharmacol 1988;25:331-40.

60. Dernellis JM, Panaretou MP. Effects of digoxin on left atrial function in heart failure. Heart 2003;89:1308-15.

61. Giunta A, Maione S, Arnese MR, et al. Effects of intravenous digoxin on pulmonary venous and transmitral flows in patients with chronic heart failure of different degrees. Clin Cardiol 1995;18:27-33.

62. Kotecha D. Magnesium for Atrial Fibrillation, Myth or Magic? Circ Arrhythm Electrophysiol 2016;9:e004521.

63. Thrall G, Lane D, Carroll D, et al. Quality of Life in Patients with Atrial Fibrillation: A Systematic Review. Am J Med 2006;119:e1-19.:448. e1-448.e19.

64. Rienstra M, Lubitz SA, Mahida S, et al. Symptoms and functional status of patients with atrial fibrillation: state of the art and future research opportunities. Circulation 2012;125:2933-43.

65. Pepine CJ. Effects of pharmacologic therapy on health-related quality of life in elderly patients with atrial fibrillation: a systematic review of randomized and nonrandomized trials. Clin Med Insights Cardiol 2013;7:1-20.

66. Hagens VE, Ranchor AV, Van Sonderen E, et al. Effect of rate or rhythm control on quality of life in persistent atrial fibrillation. results from the Rate Control Versus electrical cardioversion (RACE) Study. $J$ Am Coll Cardiol 2004;43:241-7.

67. Grönefeld GC, Lilienthal J, Kuck KH, et al. Impact of rate versus rhythm control on quality of life in patients with persistent atrial fibrillation. results from a prospective randomized study. Eur Heart $J$ 2003;24:1430-6.

68. Kotecha D, Ahmed A, Calvert M, et al. Patient-Reported Outcomes for Quality of Life Assessment in Atrial Fibrillation: A Systematic Review of Measurement Properties. PLoS One 2016;11:e0165790.

69. Kotecha D, Mohamed M, Shantsila E, et al. Is echocardiography valid and reproducible in patients with atrial fibrillation? A systematic review. Europace 2017.

70. Ziff OJ, Kotecha D. Digoxin: the good and the bad. Trends Cardiovasc Med 2016;26:585-95.

71. Dasgupta A. Impact of interferences including metabolite crossreactivity on therapeutic drug monitoring results. Ther Drug Monit 2012;34:496-506.

72. Chan AW, Tetzlaff JM, Gøtzsche PC, et al. SPIRIT 2013 explanation and elaboration: guidance for protocols of clinical trials. BMJ 2013;346:e7586.

73. Calvert M, Kyte D, von Hildebrand M, et al. Putting patients at the heart of health-care research. Lancet 2015;385:1073-4.
74. Calvert M, Kyte D, Duffy H, et al. Patient-reported outcome (PRO) assessment in clinical trials: a systematic review of guidance for trial protocol writers. PLoS One 2014;9:e110216.

75. Kyte D, Duffy H, Fletcher B, et al. Systematic evaluation of the patient-reported outcome (PRO) content of clinical trial protocols. PLoS One 2014;9:e110229.

76. Carlsson J, Miketic S, Windeler J, et al. Randomized trial of ratecontrol versus rhythm-control in persistent atrial fibrillation: the strategies of treatment of Atrial Fibrillation (STAF) study. J Am Coll Cardiol 2003;41:1690-6.

77. Hohnloser SH, Kuck KH, Lilienthal J. Rhythm or rate control in atrial fibrillation--pharmacological intervention in Atrial Fibrillation (PIAF): a randomised trial. Lancet 2000;356:1789-94.

78. Opolski G, Torbicki A, Kosior DA, et al. Rate control vs rhythm control in patients with nonvalvular persistent atrial fibrillation: the results of the polish how to treat chronic Atrial fibrillation (HOT CAFE) Study. Chest 2004;126:476-86.

79. Vora A, Karnad D, Goyal V, et al. Control of heart rate versus rhythm in rheumatic atrial fibrillation: a randomized study. J Cardiovasc Pharmacol Ther 2004;9:65-73.

80. Ware JE, Gandek B. Overview of the SF-36 Health Survey and the International Quality of Life Assessment (IQOLA) Project. J Clin Epidemiol 1998;51:903-12.

81. Gandek B, Sinclair SJ, Kosinski M, et al. Psychometric evaluation of the SF-36 health survey in Medicare managed care. Health Care Financ Rev 2004;25:5-25.

82. Herdman M, Gudex C, Lloyd A, et al. Development and preliminary testing of the new five-level version of EQ-5D (EQ-5D-5L). Qual Life Res 2011;20:1727-36.

83. Devlin NJ, Krabbe PF. The development of new research methods for the valuation of EQ-5D-5L. Eur J Health Econ 2013;14 Suppl $1: 1-3$.

84. Janssen MF, Pickard AS, Golicki D, et al. Measurement properties of the EQ-5D-5L compared to the EQ-5D-3L across eight patient groups: a multi-country study. Qual Life Res 2013;22:1717-27.

85. Spertus J, Dorian P, Bubien R, et al. Development and validation of the Atrial Fibrillation effect on QualiTy-of-Life (AFEQT) Questionnaire in patients with atrial fibrillation. Circ Arrhythm Electrophysiol 2011;4:15-25.

86. Dorian $\mathrm{P}$, Burk $\mathrm{C}$, Mullin $\mathrm{CM}$, et al. Interpreting changes in quality of life in atrial fibrillation: how much change is meaningful? Am Heart $J$ 2013;166:381-7.

87. Wynn GJ, Todd DM, Webber M, et al. The European Heart Rhythm Association symptom classification for Atrial fibrillation: validation and improvement through a Simple modification. Europace 2014;16:965-72. 